\title{
Sum of High-Risk Gene Mutation (SHGM): A Novel Attempt to Assist Differential Diagnosis for Adrenocortical Carcinoma with Benign Adenoma, Based Detection of Mutations of Nine Target Genes.
}

\author{
Guo-Yang Zheng \\ Peking Union Medical College Hospital \\ Xue-Bin Zhang \\ Peking Union Medical College Hospital \\ Han-Zhong Li ( $\nabla$ hzlipumch@163.com ) \\ Peking Union Medical College Hospital \\ Yu-Shi Zhang \\ Peking Union Medical College Hospital \\ Jian-Hua Deng \\ Peking Union Medical College Hospital \\ Xing-Cheng Wu \\ Peking Union Medical College Hospital
}

\section{Research}

Keywords: adrenocortical carcinoma, adrenocortical adenoma, differential diagnosis, gene, mutation rate

Posted Date: August 11th, 2020

DOI: https://doi.org/10.21203/rs.3.rs-54829/v1

License: (c) (i) This work is licensed under a Creative Commons Attribution 4.0 International License.

Read Full License

Version of Record: A version of this preprint was published at Biochemical Genetics on February 9th, 2021. See the published version at https://doi.org/10.1007/s10528-021-10039-w. 


\section{Abstract \\ Background}

There has been no research on applying gene detection to differential diagnosis of adrenocortical carcinoma (ACC). We attempted to explore a novel auxiliary method for differential diagnosis between ACC with benign adrenocortical adenoma (ACA), based on mutations of target genes in tissues.

\section{Methods}

Nine genes were chosen as target genes, including TP53, CTNNB1, ARMC5, PRKAR1A, ZNRF3, RB1, APC, MEN1 and RPL22. Exons capture and sequencing of target genes were performed in 98 cases of tissue samples by using FastTarget technology, including 41 ACC tissues, 32 ACA tissues and 25 normal adrenal gland tissues. Significant mutations were detected and identified, and the clinical information was collected, for further comparative analysis and application to assist differential diagnosis of ACC.

\section{Results}

We identified 132 significant gene mutations and 227 significant mutation sites in 37 ACC tissues, much more than ACA and normal adrenal gland tissues. Mutation rates of 6 genes in ACC tissues were obviously higher than ACA tissues, including ZNRF3, ARMC5, TP53, APC, RB1, and PRKAR1A, which were regarded as high-risk genes. The sum of mutated high-risk genes detected in each sample was denominated sum of high-risk gene mutation (SHGM), and the rates of SHGM $>0$ and SHGM $>1$ in ACC tissues were $73.0 \%$ and $62.2 \%$ respectively, which were both obviously higher than those in ACA tissues, with significant statistic differences. Especially for the 8 cases of ACC with diameter $<5 \mathrm{~cm}, \mathrm{SHGM}>0$ and SHGM > 1 were found in 6 samples (75\%) and 4 samples (50\%), respectively. However, no relevance was found between SHGM and clinical characteristics of ACC.

\section{Conclusions}

We identified 6 high-risk genes in ACC tissues, of which the mutation rates in ACC tissues were significantly higher than those in ACA or normal adrenal gland tissues. The sum of mutated high-risk genes detected in ACC tissues was denominated SHGM, which was potential to assist the differential diagnosis of ACC with ACA, especially for the small-size ACC.

\section{Background}

Adrenocortical carcinoma (ACC) is a rare epithelial malignant tumor clinically, derived from adrenal cortical cells with a low annual incidence rate of approximately $0.7-2$ people per million ${ }^{[1]}$. Tumor stage is an important factor affecting the prognosis of ACC patients. Researches showed patients with stage I-II 
commonly survive for more than 5 years, while the overall survival of ACC patients with stage III was generally 3-5 years. The worst survival prognosis appeared in patients with stage IV, which usually survived merely for less than 1 year ${ }^{[2-5]}$. Surgical resection of tumor is recommended as the preferred therapy for ACC, and the completeness of resection is another important influence factor of prognosis of $\operatorname{ACC}^{[6,7]}$.

In clinical practice, it is sometimes difficult to differentiate ACC from benign adenoma, especially for small-size tumors, which may lead to inadequate surgical resection and poor survival prognosis. Unfortunately, present pathological examinations including Weiss system and Ki-67 index, is not infallible for the differential diagnosis of $A C C^{[8]}$, so some ACC patients misdiagnosed as benign adrenocortical adenoma (ACA) before operations might still miss the opportunities of postoperative salvage therapy to improve prognosis.

Therefore, we believe it is still necessary to explore novel differential diagnosis methods for ACC, at least an auxiliary method of current diagnosis system. So far, there has been no genetic methods for differential diagnosis of ACC in clinical practice. In this research, we screened out nine genes associated with ACC as target genes according to the present data of genetic researches, and detected mutations of target genes in tissue samples by using FastTarget exons capture and sequencing technology. Then we aimed to explore a novel auxiliary method of differential diagnosis for ACC, by comparing and analyzing the mutation characteristics of target genes between ACC tissues with benign ACA tissues.

\section{Methods}

\section{Object}

We took totally 98 cases of formalin fixed and paraffin embedded tissue samples as research subject, including 41 cases of ACC (C1-C41), 32 cases of ACA (A1-A32) and 25 cases of normal adrenal gland tissues (N1-N25), obtained from the patients receiving operations in Peking Union Medical College Hospital (PUMCH) from April 2003 to January 2018. All samples were performed target exons capture and sequencing of 9 target genes by using FastTarget technology. All patients were diagnosed by pathological results and clinical follow-up, and two patients (C4 and C19) misdiagnosed as benign ACA by initial pathological examinations were finally diagnosed as ACC until the tumor recurred after surgery. All patients had signed the informed consent forms, and the clinical information was collected, including age, gender, tumor diameter, tumor stage, endocrine function and Ki-67 index. Tumor diameter was measured by pathological examinations, and tumor stage was based on ENSAT staging criteria. The research was approved by the ethics committee of our institute.

\section{Design}

\section{All Exons sequencing of 9 target genes}


Based on the present researches on genic characterization of adrenocortical carcinoma, we chose nine genes correlated with ACC as target genes for the exons capture and sequencing in this study, including TP53, CTNNB1, ARMC5, PRKAR1A, ZNRF3, RB1, APC, MEN1 and RPL22 ${ }^{[9,10]}$. Exons capture and enrichment of above target genes in all samples were performed by using PCR technology, and Illumina MiSeq Benchtop Sequencer and analysis software were used to detect and screen out significant mutations occurring in samples. Mutations of target genes in ACC tissues and other tissues were performed further comparative analysis and clinical correlation analysis, in order to explore auxiliary method for the differential diagnosis of ACC.

\section{Main instruments and reagents}

Main instruments and reagents involved in this study were demonstrated in Table 1.

Table 1

Main instruments and reagents

\begin{tabular}{|ll|}
\hline Instruments & Origin \\
\hline Applied Biosystems 2720 Thermal Cycler & Applied Biosystems, USA \\
\hline 3730xl Genetic Analyzer & Thermo Fisher Scientific,USA \\
\hline Eppendorf 5810R Centrifuge & Eppendorf,Germany \\
\hline NanoDrop 2000 Spectrophotomete & NanoDrop technologies, USA \\
\hline Invitrogen Qbit Spectrophotometer & Invitrogen,USA \\
\hline MiSeq Benchtop Sequencer & Illumina,USA \\
\hline Reagents & Origin \\
\hline HotstarTaq DNA Polymerase Kit & QIAGEN,Germany \\
\hline Q5 DNA polymerase Kit & New England Biolabs,USA \\
\hline GeneScan-500 Liz Size Standard & Thermo Fisher Scientific,USA \\
\hline Agencourt AMPure XP-PCR Purification & Beckman,USA \\
\hline NextSeqReagent Kit v3 & Illumina,USA \\
\hline
\end{tabular}

\section{Sequencing procedure}

a. $1 \%$ agarose electrophoresis was used for DNA quality test.

b. Primers design of the target genes and multiple polymerase chain reaction (PCR) primer panel optimization according to the standard of 20 pairs of primers for each panel.

c. Multiple PCR amplification was performed taking sample genome as template, and specific tag sequences were added into the mixture of PCR amplification products from the same sample. 
d. Finally, the FastTarget sequencing library obtained from amplified PCR products of all samples, were sequenced on the Illumina MiSeq platform with $2 \times 150 \mathrm{BP} / 2 \times 250 \mathrm{BP}$ double terminal sequencing mode to obtain FastQ data.

\section{Bioinformatic analysis and mutation annotation}

a. Original FastQ data file obtained by Illumina high-throughput sequencing platform was performed quality assessment by FastQC software (http://www.bioinformatics. babraham.ac.uk/projects/fastqc), in order to draw the distribution diagram of base error rate, base quality and base composition of original data.

b. All the Reads were sequenced twice in forward and reverse (R1 and R2), and then were performed sequence calibration using FLASH (Fast length adjustment of short reads to improve genome assemblies) software (http://www.cbcb.umd.edu/ software/flash), getting the combined FastQ file of R1 and R2. FastX software (http://hannonlab.cshl.edu/fastx_toolkit/index.html) was used to control the quality of sequences covering target regions.

c. BWA software (http://bio-bwa.sourceforge.net/) was used to compare original sequencing data with human genome data (USCS hg19 version, http://hgdownload.soe.ucsc.edu/goldenPath/hg19/bigZips/), and the comparison results were statistically analyzed by Picard (https://broadinstitute.github.io/picard/).

d. Preliminary comparison results obtained by BWA software were calibrated by adopting the GATK standard process (https://software.broadinstitute.org/gatk/best-practices/), in order to improve the accuracy of identification of SNVs and indels, greatly reducing the false-positive and false negative produced in the process of sequencing and comparison.

e. We used two methods to sequence single nucleotide variants (SNVs) and insertion/ deletion (Indel) of all samples respectively, which were VarScan (http://varscan. sourceforge.net/) and GATK Haplotype Caller (https://software.broadinstitute. org/gatk/ best-practices/). We compared the data of detected mutations with latest released exons sequencing data of normal population database, functional database and disease database, by using ANNOVAR software (http://annovar.openbioinformatics. org/en/latest/). The high-frequency mutations in healthy human and synonymous mutations were removed, in order to discover mutations with biological significance.

\section{Statistical Analysis}

All statistical analysis was conducted by using SPSS software (Statistical Product and Service Solutions version 24.0, USA). The data was described in the form of mean ( \pm standard deviation), median (range) or rate. Results of enumeration data were compared using Chi-squared test and Fisher's exact test. Results of measurement data were compared by nonparametric test. Statistical significance was considered as $p$ $\leq 0.05$.

\section{Results}




\section{Quality control}

We eliminated 4 unqualified samples (C3, C5, C7, C17), in which we failed to obtain the amplifications of target gene fragments. All the other 94 samples were available to obtain enough qualified amplifications of target genes by next-generation sequencing (NGS), which could be used for further bioinformatic analysis (Fig. 1).

We used BWA software to compare original sequencing data of all samples with human genome data, which was used as reference genome. Quality analysis of sequencing showed that, sequencing depth of all samples ranged from $190 \mathrm{X}$ to $1536 \mathrm{X}$, and the mean was 733X (Fig. 2). Sequencing depth of target gene fragments was above $10 \mathrm{X}$ in $95.1 \%$ samples (Fig. 3), which was above $20 \mathrm{X}$ and $30 \mathrm{X}$, in $92 \%$ and $89 \%$ samples, respectively. In addition, quality scores and sequence content across all bases were also performed. In conclusion, results of quality analysis indicated that the results of sequencing were reliable.

\section{Overview of sequencing results of target gene mutations}

We sequenced and analyzed mutation information of 9 target genes in 94 tissue samples, including 37 ACC tissue samples, 32 ACA tissue samples and 25 normal adrenal gland tissue samples, using FastTarget technology to perform target exons capture sequencing. We selected nine genes as target genes for exons capture and sequencing, which were TP53, CTNNB1, ARMC5, PRKAR1A, ZNRF3, RB1, APC, MEN1 and RPL22, including 202 PCR sequencing fragments in all.

We used VerScan and GATK HaplotypeCaller two methods to sequence single nucleotide variants (SNVs) and insertion/ deletion (Indel) of all samples respectively. Totally, we detected 3017 exonic mutations, 3732 intronic mutations, 458 splicing mutations, 317 5'-UTR (untranslated region) mutasions and 39 3'UTR mutasions of target genes in 94 tissue samples. Focusing solely on exonic mutations, we detective 1062 nonsynonymous single nucleotide variants (SNVs), 1830 synonymous SNVs, 27 stopgain mutations, 1 stoploss mutation, 95 frameshift deletion and 1 nonframeshift insertion mutation, without nonframeshift deletion or frameshift insertion detected. Because some mutations were repeatedly detected in multiple tissue samples, we finally identified 505 kinds of exonic mutations and 50 kinds of splicing mutations, which were available detective in one or more tissue samples. Then we targeted the nonsynonymous SNVs, stopgain, stoploss, frameshift deletion and splicing mutaions as analysis subjects.

Due to the lacking of normal tissues in the same patients for comparative analysis, we performed comparative analysis of all detected mutations by using ANNOVAR, and compare these mutation data with exons sequencing data of 300 normal tissues additionally. Then we removed normal mutations, high-frequency mutations in healthy human, and synonymous mutations, in order to discover mutations with biological significance. Finally, we identified that 178 gene mutations with biological significance occurred in the 94 tissue samples, with 280 significant mutation sites detected in all. 
In 37 ACC tissue samples, 132 significant gene mutations and 227 significant mutation sites were identified, including 169 nonsynonymous SNVs, 19 stopgain mutations, 1 stoploss mutation, 19 frameshift deletions, and 19 splicing mutations. The median number of target gene mutations and mutation sites occurring in ACC samples were 4.0 (1.5-6.5) and 4.0 (2.0-10.0), respectively. The maximum of gene mutations was 8 , detected in sample C35, and the maximum of mutation sites was 19 , detected in sample C11. In 32 samples of ACA group, median number of gene mutations and mutation sites were $1.0(0-1.0)$ and $1.0(0-2.0)$, respectively. And the counterparts in 25 normal adrenal gland tissues were both $1.0(0-1.0)$ (Table 2). The statistical results showed the amount of target gene mutation and mutation sites detected in adrenocortical carcinoma tissues were both higher than those in adrenocortical adenoma tissues and normal adrenal gland tissues, with significant difference $(P<0.001)$ (Fig. 4). This results indicated that exons sequencing of nine target genes in our research may be potential to assist distinguishing ACC from ACA or normal adrenal gland. 
Table 2

Overview of mutations of target genes

\begin{tabular}{|llll|}
\hline & ACC & ACA & Normal gland \\
& $\mathbf{n = 3 7}$ & $\mathbf{n = 3 2}$ & $\mathbf{n = 2 5}$ \\
\hline Sum of gene mutations & 132 & 30 & 16 \\
\hline MEN1 & 20 & 14 & 10 \\
\hline ZNRF3 & 20 & 2 & 1 \\
\hline ARMC5 & 19 & 2 & 1 \\
\hline TP53 & 19 & 2 & 2 \\
\hline CTNNB1 & 13 & 7 & 0 \\
\hline APC & 12 & 1 & 2 \\
\hline RB1 & 11 & 0 & 0 \\
\hline PRKAR1A & 9 & 0 & 0 \\
\hline RPL22 & 9 & 2 & 0 \\
\hline Number of mutated genes, Median (range) & $4.0(1.5-6.0)$ & $1(0-1)$ & $1(0-1)$ \\
\hline Sum of mutation sites & & $\mathbf{P}<0.001$ & $\mathbf{P}<0.001$ \\
\hline nonsynonymous SNV & 227 & 35 & 18 \\
\hline frameshift deletion & 19 & 21 & 13 \\
\hline frameshift insertion & 169 & 10 & 3 \\
\hline stopgain & 19 & 0 & 0 \\
\hline stoploss & 0 & 0 & 0 \\
\hline splicing & 19 & $\mathbf{P}$ & 0 \\
\hline Number of mutation sites, Medan (range) & $4(2-10)$ & $1(0-2)$ & $1(0-1)$ \\
\hline & & $\mathbf{P}<0.001$ \\
\hline
\end{tabular}

\section{Comparative analysis of target gene mutation in ACC}

Although in 6 samples (C2, C19, C26, C28, C30 and C33) we failed to detect any significant gene mutations, 132 significant gene mutations and 227 mutation sites were identified in other 31 ACC samples, by exons capture and sequencing of nine target genes. The mutation rates of the nine target genes in ACC tissue samples from high to low were: 54.1\% (20/37) of MEN1, 54.1\% (20/37) of ZNRF3, 
$51.4 \%$ (19/37) of ARMC5, 51.4\% (19/37) of TP53, 35.1\% (13/37) of CTNNB1, 32.4\% (12/37) of APC, $29.7 \%(11 / 37)$ of RB1, 24.3\% (9/37) of PRKAR1A, and 24.3\% (9/37) of RPL22. In the 227 mutation sites, the incidences of each type of mutation were: $74.4 \%$ (169/227) of nonsynonymous SNV, 8.4\% (19/227) of stopgain mutations, $8.4 \%$ (19/227) of frameshift deletions, $8.4 \%(19 / 227)$ of splicing mutations, and $0.4 \%(1 / 227)$ of stoploss mutation. The main mutation type of RPL22 gene occurring in ACC tissues was frameshift deletion $(66.7 \%, 6 / 9)$, while the main mutation type of the other genes was nonsynonymous SNV. The mutation information of nine target genes in ACC was showed in Fig. 5.

Comparative analysis of mutations of the nine target genes between ACC tissues with ACA tissues, demonstrated that mutation rates of 6 genes in ACC group were obviously higher than those in ACA group, including ZNRF3, ARMC5, TP53, APC, RB1, and PRKAR1A. As for the other 3 genes (MEN1, CTNNB1, and RPL22), there was no difference between ACC and ACA in mutation rate or mutation type (Table 3). The above result of analysis suggested that mutations of ZNRF3, TP53, ARMC5, APC, RB and PRKAR1A may be potential to assist the differential diagnosis between ACC and ACA. Comparative analysis between ACC and normal adrenal gland showed that mutation rates of 7 genes in ACC tissues were higher than those in normal tissues, which were ZNRF3, ARMC5, TP53, CTNNB1, APC, RB1 and PRKAR1A (Table 4). Mutation information of nine target genes in ACC, ACA, and normal adrenal gland tissues were demonstrated in Fig. 5 - Fig. 7, respectively.

Table 3

Mutation rates and types of target genes in ACC and ACA tissues

\begin{tabular}{|lllllll|}
\hline Gene & \multicolumn{2}{l}{ No. of gene mutation (rate) } & \multicolumn{3}{l|}{$\begin{array}{l}\text { Type of mutation } \\
\text { (nonsynonymous SNV / Sum) }\end{array}$} \\
\cline { 2 - 7 } & $\begin{array}{l}\text { ACC } \\
\text { (n=37) }\end{array}$ & $\begin{array}{l}\text { ACA } \\
(\mathbf{n}=32)\end{array}$ & $\mathbf{P}$ & ACC & ACA & P \\
& 20(54.1\%) & $14(43.8 \%)$ & 0.472 & $23 / 32$ & $10 / 18$ & 0.352 \\
\hline MEN1 & $20(54.1 \%)$ & $2(6.3 \%)$ & $<\mathbf{0 . 0 0 1}$ & $19 / 28$ & $1 / 2$ & 1.000 \\
\hline ZNRF3 & $19(51.4 \%)$ & $2(6.3 \%)$ & $<\mathbf{0 . 0 0 1}$ & $36 / 43$ & $2 / 2$ & 1.000 \\
\hline ARMC5 & $19(51.4 \%)$ & $2(6.3 \%)$ & $<\mathbf{0 . 0 0 1}$ & $17 / 25$ & $0 / 2$ & 0.128 \\
\hline TP53 & $13(35.1 \%)$ & $7(21.9 \%)$ & 0.291 & $22 / 26$ & $7 / 8$ & 1.000 \\
\hline APC & $12(32.4 \%)$ & $1(3.1 \%)$ & $\mathbf{0 . 0 0 2}$ & $29 / 34$ & $1 / 1$ & 1.000 \\
\hline RB1 & $11(29.7 \%)$ & 0 & $\mathbf{0 . 0 0 1}$ & $12 / 17$ & - & - \\
\hline PRKAR1A & $9(24.3 \%)$ & 0 & $\mathbf{0 . 0 0 3}$ & $8 / 13$ & - & - \\
\hline RPL22 & $9(24.3 \%)$ & $2(6.3 \%)$ & 0.052 & $3 / 9$ & $0 / 2$ & 1.000 \\
\hline Total & 132 & 30 & - & $169 / 227$ & $21 / 35$ & 0.075 \\
\hline
\end{tabular}


Table 4

Mutation rates and types of target genes in ACC and normal tissues

\begin{tabular}{|lllllll|}
\hline Gene & \multicolumn{2}{l}{ No. of gene mutation (rate) } & \multicolumn{4}{c|}{ Type of mutation } \\
& & & \multicolumn{5}{c|}{ nonsynonymous SNV / Sum } \\
\cline { 2 - 7 } & ACC & Normal & P & ACC & Normal & P \\
& $(\mathbf{n}=37)$ & $(\mathbf{n = 2 5 )}$ & & & & \\
\hline MEN1 & $20(54.1 \%)$ & $10(40.0 \%)$ & 0.311 & $23 / 32$ & $9 / 12$ & 1.000 \\
\hline ZNRF3 & $20(54.1 \%)$ & $1(4.0 \%)$ & $<0.001$ & $19 / 28$ & $1 / 1$ & 1.000 \\
\hline ARMC5 & $19(51.4 \%)$ & $1(4.0 \%)$ & $<0.001$ & $36 / 43$ & $1 / 1$ & 1.000 \\
\hline TP53 & $19(51.4 \%)$ & $2(8.0 \%)$ & $<0.001$ & $17 / 25$ & $0 / 2$ & 0.128 \\
\hline CTNNB1 & $13(35.1 \%)$ & 0 & $\mathbf{0 . 0 0 1}$ & $22 / 26$ & - & - \\
\hline APC & $12(32.4 \%)$ & $2(8.0 \%)$ & $\mathbf{0 . 0 3 1}$ & $29 / 34$ & $2 / 2$ & 1.000 \\
\hline RB1 & $11(29.7 \%)$ & 0 & $\mathbf{0 . 0 0 2}$ & $12 / 17$ & - & - \\
\hline PRKAR1A & $9(24.3 \%)$ & 0 & $\mathbf{0 . 0 0 3}$ & $8 / 13$ & - & - \\
\hline RPL22 & $9(24.3 \%)$ & 0 & 0.052 & $3 / 9$ & - & - \\
\hline Total & 132 & 16 & - & $169 / 227$ & $13 / 18$ & 0.835 \\
\hline
\end{tabular}

\section{SHGM to assist differential diagnosis of ACC with ACA}

We proposed a novel concept that was denominated "sum of high-risk gene mutation", abbreviated to SHGM, as an auxiliary method for differential diagnosis of ACC with ACA, by exons capture and sequencing of 9 target genes designed in our research. We elected six genes as high-risk mutant genes related to ACC, which were ZNRF3, TP53, ARMC5, APC, RB1, PRKAR1A, based on the above analysis results. Mutation rates of high-risk genes in ACC tissues were much higher than those in ACA tissues, and we count the sum of mutated high-risk genes detected in each sample, as the SHGM of the sample.

Then we calculated the SHGM of every samples in ACC group and ACA group. For the 37 samples of ACC tissues, SHGM > 0 was found in 27 samples (73.0\%), and SHGM > 1 was identified in 23 samples (62.2\%). However, we found SHGM $>0$ in only 6 samples (18.8\%) of ACA group, and there was only 1 sample (A9) with $\mathrm{SHGM}>1$ (3.1\%). The rates of SHGM $>0$ and $\mathrm{SHGM}>1$ in ACC tissues were both obviously higher than those in ACA tissues, with significant statistic differences (Table $5-1$ ). More importantly, in the 8 tissue samples of ACC with diameter less than $5 \mathrm{~cm}$, which tended to be misdiagnosed as ACA, we found SHGM $>0$ in 6 samples (75\%) and SHGM > 1 in 4 samples (50\%) (Table $5-2)$. It indicated that the SHGM might contribute to differential diagnosis between ACC with ACA in some degree, especially for the 
diagnosis of ACC with small diameter. If we set SHGM $>0$ as reference standard to detect the efficacy of differential diagnosis between ACC with ACA tissue samples included in our research, the specificity and sensibility of identification of ACC were $72.2 \%$ and $81.8 \%$ respectively. If the reference standard was set as $\mathrm{SHGM}>1$, the specificity and sensibility were $68.9 \%$ and $95.8 \%$ respectively.

Table 5-1.

Sum of high-risk gene mutation (SHGM) in ACC vs ACA

\begin{tabular}{|llll|}
\hline & ACC Group & ACA Group & P \\
\hline SHGM, median (range) & $2(2-4)$ & $0(0-0)$ & $<0.001$ \\
\hline Rate of SHIRMS $>0$ & $73.0 \%(27 / 37)$ & $18.8 \%(6 / 32)$ & $<0.001$ \\
\hline Rate of SHIRMS $>1$ & $62.2 \%(23 / 37)$ & $3.1 \%(1 / 32)$ & $<0.001$ \\
\hline
\end{tabular}

Table 5-2.

Sum of high-risk gene mutation (SHGM) in ACC with diameter $\leq 5 \mathrm{~cm}$

\begin{tabular}{|lllllllll|}
\hline Sample Nomber & C1 & C4 & C11 & C19 & C22 & C29 & C37 & C40 \\
\hline Diameter, cm & 5.0 & 3.8 & 4.5 & 4.0 & 3.0 & 2.0 & 4.7 & 4.5 \\
SHGM & 2 & 3 & 6 & 0 & 0 & 5 & 1 & 1 \\
ZNRF3 & - & + & + & - & - & + & - & - \\
TP53 & + & + & + & - & - & + & - & - \\
ARMC5 & + & - & + & - & - & + & - & + \\
APC & - & + & + & - & - & + & + & - \\
RB1 & - & - & + & - & - & - & - & - \\
PRKAR1A & - & - & + & - & - & + & - & - \\
\hline
\end{tabular}

We also performed correlation analysis between SHGM and clinical characteristics of ACC, including gender, age, endocrine function, diameter, tumor stage and Ki-67 index. Unfortunately, we did not find any relevance between SHGM and above characteristics (Table 6), which indicated SHGM might be incapable of predicting clinical characteristics or prognosis of ACC. 
Table 6

Correlation analysis between SHGM with clinical characterisics

\begin{tabular}{|llll|}
\hline Clinical characteristics & Result & correlation with SHGM & $\mathbf{P}$ \\
\hline Age, years, mean \pm SD $(95 \% \mathrm{Cl})$ & $52.5 \pm 9.5$ & None & 0.888 \\
\hline Diameter, cm, mean \pm SD $(95 \% \mathrm{Cl})$ & $5.8 \pm 1.1$ & None & 0.213 \\
\hline Ki-67 index (\%), median (range) & $20(10-25)$ & None & 0.593 \\
\hline Clinical characteristics & $\mathbf{n}(\%)$ & SHGM & $\mathbf{P}$ \\
\hline Sex & & & 0.419 \\
male & $12(32.4 \%)$ & $3.5(0.25-4.75)$ & \\
female & $25(67.6 \%)$ & $2(0-3.5)$ & 0.631 \\
Endocrine function & & & 0.240 \\
non-function & $17(45.9 \%)$ & $3(0-5)$ & \\
function & $20(54.1 \%)$ & $2(1-3.5)$ & \\
\hline Tumor stage & & & \\
stage I-II & $19(51.4 \%)$ & $2(0-4)$ & \\
stage III-IV & $18(48.6 \%)$ & $3(0.75-5)$ & \\
\hline
\end{tabular}

\section{Discussion}

\section{Importance and limitation of pathological differential diagnosis of ACC}

Adrenocortical carcinoma is a rare malignant tumor with a low incidence and relatively poor prognosis ${ }^{[1}$,

11]. Complete resection of tumor and surrounding tissues is considered as the optimal treatment of ACC, and the completeness of surgical resection is an important influencing factor on the prognosis of ACC ${ }^{[12]}$. Complete resection range should include at least the whole tumor with complete capsule, the whole adrenal gland, and as much surrounding adipose tissue as possible. Research has shown that postoperative adjuvant therapy, such as radiotherapy or mitotane treatment, might contribute to improving the postoperative prognosis of ACC, especially for those tumors resected with positive margin ${ }^{[7,13]}$. Unfortunately, some adrenocortical carcinoma with small tumor size may tend to be misdiagnosed as benign adrenocortical adenoma, which may mislead surgeons and lead to incomplete tumor resection scope. Therefore, accurate differential diagnosis is important for ACC patients to choose appropriate therapy strategy. Pathological diagnosis is the most important defining characteristics for differential diagnosis of ACC, which could help deciding the requirement of postoperative adjuvant therapy and guiding the follow-up strategy. More importantly, for the ACC patients who were 
misdiagnosed as benign ACA and received incomplete tumor resection, accurate postoperative pathological diagnosis could warn the patients to perform rescue therapy as soon as possible, such as secondary surgery, mitotane therapy, or radiotherapy, in order to improve the prognosis of patients.

However, in clinical practice, pathological diagnosis is not entirely reliable to distinguish ACC from ACA, especially for the differential diagnosis of small-size tumors with diameter less than $5 \mathrm{~cm}$. Cytological characteristics of ACC tissues is similar with ACA tissues under the microscope sometimes, due to the endocrine function of adrenocortical tumors ${ }^{[8]}$. Cells of benign adenoma with endocrine function may appear cytological characteristics similar to malignant ACC, such as caryogram, pathological mitosis, and cell ultrastructure including the change of mitochondria, basement membrane, or endoplasmic reticulum. So far, there have been not particular specific immunohistochemical markers for accurate identification of ACC. Current reference immunohistochemical markers clinically used for differential diagnosis of ACC, merely could indicate whether the tumor derived from adrenal cortex or not ${ }^{[14-17]}$. Those markers, such as a-inhibin, synaptophysin, melanA, calretinin, SF1 and so on, were mainly used to distinguish adrenocortical tumors from adrenal pheochromocytomas, adrenal metastases, or other types of retroperitoneal neoplasms, while could not be regarded specific markers for ACC.

Weiss system is regarded as the most effective and widely-used clinical diagnostic system for adrenocortical carcinoma at present. In Weiss system there are 9 criteria in total, and the presence of 3 or more criteria will suggest making the diagnosis of ACC, with sensitivity and specificity both more than $90 \%{ }^{[18]}$. Ki-67 index is another important diagnostic criterion for differential diagnosis of ACC in the clinical practice ${ }^{[19]}$. It is generally considered that $\mathrm{Ki}-67$ index more than $5 \%$ is probably associated with $A C C$, and the higher index will indicate the worse prognosis, such as overall survival and progression free survival ${ }^{[20]}$. In addition, destroy of intracellular reticular fibers observed by cytoskeleton staining could be also regarded as another differential criterion for ACC ${ }^{[21,22]}$. Unfortunately, differential diagnosis of ACC only based on the above pathological methods may be not entirely reliable. In clinical practice, a few patients pathologically diagnosed with benign ACAs finally proved to be malignant ACCs, and might miss the opportunity of radical treatment or rescue therapy. Similarly, a few patients pathologically diagnosed with ACC finally did not appear as malignant biological properties.

Therefore, it is still necessary and valuable to explore another novel identification method to assist the differential diagnosis of adrenocortical carcinoma. In consideration of the current limitation of pathological diagnostic methods and the absence of genetic diagnostic methods, we aimed to explore a novel differential diagnosis system based on the condition of gene mutations in ACC tissues. We expected to improve the accuracy of differential diagnosis for ACC, and contribute to improve the prognosis of ACC patients who were misdiagnosed as benign ACA, by using this novel genetic diagnostic method as supplement or combination of current diagnosis methods such as Weiss system and Ki-67 index. Then, the ACC patients misdiagnosed as benign tumor before operation could receive timely salvage therapy in order to improve survival prognosis 


\section{Current status of genetic researches of adrenocortical carcinoma.}

Some past researches have demonstrated that mutations of TP53 gene, CTNNB1 gene, MEN1 gene and CDKN2A gene might be associated with $\mathrm{ACC}^{[23-28]}$. There were two convincing researches published on prestigious journals recently, which provided more reliable data of genetic researches in $\mathrm{ACC}^{[9-10]}$. A research published in Journal Nature Genetics in 2014 performed whole exons sequencing on 45 cases of ACC tissues ${ }^{[9]}$. The research identified several mutant genes associated with ACC, such as ZNRF3, CTNNB1, TP53, CDKN2A, RB1 and MEN1. In addition, it discovered that copy number variations of some genes were also related with ACC, such as amplification of TERT gene (5p. 15.33), and homozygous deletion of CDKN2A (9p21.3), RB1 (13q14) and ZNRF3 (22q12.1). Another famous research published in Journal Cell in 2016, detected totally 8814 somatic single-nucleotide variants (SNVs) and small indels mutations, including 6664 non-synonymous mutations and 2150 synonymous mutations ${ }^{[10]}$. This study identified several significant mutant genes in ACC tissues, including TP53, CTNNB1, MEN1, PRKAR1A and RPL22. Increased copy numbers of TERT gene (5p15.33), TERF2 gene (16q22.1), CDK4 gene (12q14.1) and CCNE1 gene (19q12), as well as deletions of RB1 gene (13q14.2), CDKN2A gene (9p21.2) and ZNRF3 gene (22q12.1), were also detected in ACC tissues.

According to the data of above researches, ZNRF3 gene mutation was newly discovered associated with ACC, with a mutation rate of approximately $21 \%$ according to above researches. ZNRF3 gene encodes transmembrane E3 ubiquitin ligase on cell surface, which could inhibit function of Wnt/ $\beta$-catenin signaling pathway, so mutation of ZNRF3 could result in the over-activation of this signaling pathway. Besides, mutations of CTNNB1, MEN1 and APC also could cause over-activation of Wnt/ $\beta$-catenin pathway, and mutation rates of above genes detected in ACC were approximately $20 \%, 7 \%$ and $3 \%$, respectively. The $W n t / \beta$-catenin signaling pathway was involved in regulating cell adhesion, proliferation, apoptosis and other cellular functions, which was usually found over-activated in ACC tissues. TP53/Rb was another signaling pathway associated with ACC, which could be influenced by mutation of gene TP53 and Rb1. TP53 was located in chromosome 17 p13, involved in cell proliferation and apoptosis, and the mutation rate of TP53 detected in ACC was reported as $21.3 \%$. In addition, PRKAR1A, RPL22 and NF1 were newly discovered genes related to ACC recently, and mutation rates detected in ACC were $8 \%$, $7 \%$ and $5 \%$, respectively. The mutation of ARMC 5 gene was frequently detected in primary macronodular adrenal hyperplasia, but there has been no research detecting mutation status of ARMC5 in ACC tissues $^{[29]}$.

According to the above research data, we finally selected 9 genes associated with ACC as target genes, which were ZNRF3, TP53, CTNNB1, PRKAR1A, MEN1, RB1, APC, RPL22 and ARMC5, in order to explore mutation characteristics and clinical value of the target genes in ACC tissues. Then we expected to apply it to the auxiliary differential diagnosis of ACC after operations, in order to improve the prognosis of preoperative misdiagnosed ACC patients, by timely salvage therapy such as secondary surgery, radiotherapy and mitotane treatment. 


\section{Clinical significance and limitations of SHGM}

In this research, we analyzed the mutation rates and types of the 9 target genes in ACC tissues, by using exons capture enrichment sequencing, and compared the data with that of ACA and normal adrenal gland tissues. The results showed that the mutation rates of 6 genes detected in ACC tissues were significantly higher than those in ACA and normal adrenal gland tissues, which were ZNRF3, TP53, ARMC5, APC, RB1 and PRKAR1A. We regarded the 6 genes as high-risk genes associated with ACC, and proposed a novel concept named sum of high-risk mutation genes (SHGM), defined as the count of mutations of above 6 high-risk genes detected in ACC tissues. We applied SHGM to the auxiliary differential diagnosis of ACC, which is the innovation of this research. In the 37 cases of ACC tissues, SHGM > 0 was identified in 27 samples (73.0\%), and SHGM > 1 was identified in 23 samples $(62.2 \%)$. While in the 32 cases of ACA tissues, there were only 6 samples with SHGM $>0$ (18.8\%) and only one sample with SHGM $>1(3.1 \%)$, and the difference with ACC group was statistically significant $(P<0.05)$. The results indicated that the SHGM may be helpful in the differential diagnosis of adrenocortical carcinoma and benign adenoma. If we set SHGM $>1$ as reference index for diagnosis of ACC, the sensibility for identification of ACC could be up to $95.8 \%$. However, the specificity was only $68.9 \%$, which indicated that come benign ACA patients might be overestimated as malignant ACC, leading to the possibility of over-treatment. Therefore, we expected SHGM to be an auxiliary diagnostic index, which should be applied to the differential diagnosis of ACC together with clinical existing diagnostic methods, such as Weiss system, Ki-67 index and cellular reticular fiber structure staining, in order to maximize the diagnostic accuracy.

More importantly, we found that SHGM might be more helpful and valuable in the differential diagnosis of small-size ACC with benign adenoma, reflecting the clinical significance and innovation of the establishment of SHGM. In the 8 cases of ACC with tumor diameters $\leq 5 \mathrm{~cm}$, SHGM $>0$ was identified in 6 cases $(75 \%)$, and the rate of SHGM $>1$ was $50 \%$. Among the 8 cases, there were two patients (C4 and C19) misdiagnosed as benign ACA by postoperative pathological diagnosis. Unfortunately, the tumors recurred rapidly in the two patients due to the absence of timely salvage treatment after operations, with DFS of 9.6 months for C4 and 16.0 months for C19, respectively. While, researches showed the survival of small-size adrenocortical carcinoma were usually more than 5 years ${ }^{[2]}$, much better than the surviving for approximately 3 years of the above 2 patients. The SHGM detected in C4 and C19 were 3 and 0 respectively, so the application of SHGM for differential diagnosis could have improved the prognosis of C4 patient by timely salvage therapy after operation. Therefore, we considered that the SHGM was helpful for the auxiliary differential diagnosis of ACC after surgery and valuable to improve the clinical prognosis of ACC patients, especially for the small-size tumors with diameters $\leq 5 \mathrm{~cm}$, which might be tended to be misdiagnosed as benign tumor.

It is inevitable that there are some limitations in this research. Firstly, the sequencing data of tumor tissue lacked the control of normal tissue of the same patient, because the ACC samples were obtained from formalin fixed and paraffin embedded tissues of patients who had received operations in our hospital in the past. Most of the patients have died or lost contact, so we were unable to obtain the normal tissues 
from the same patients as control to screen significant mutant genes and mutation sites. Then we compared the sequencing results with the present data of whole exon and genome sequencing results in normal population, which might be one of the reasons why the mutation rates of the target genes in our research were a little higher than those of the corresponding genes published in past studies, and the other possible reasons might include racial differences and the differences of sequencing platforms or sequencing technology. All tissue samples in our study were compared with the same database, and the results of quality control showed the amplifications of target genes fragments were qualified. We believed the results of our research were reliable and clinically significant, since the original intention was expecting to apply SHGM to auxiliary differential diagnosis of ACC with ACA, rather than exploring the pathogenic mutant genes and mutation sites of adrenocortical carcinoma. Secondly, the SHGM system only including 6 target genes based on the sequencing and analysis results in this study, and we believe there are still some genes with differential diagnosis significance for ACC to be found and studied. At last, we failed to find any significant statistic correlations between SHGM with other clinical characteristics of ACC, including age, gender, tumor diameter, tumor stage, endocrine function and Ki-67 index, so we do not recommend the SHGM for the evaluation of clinical features and prognosis of ACC.

\section{Conclusions}

In this research, we performed target exons capture and sequencing of 9 target genes in ACC, ACA and normal adrenal gland tissues. Analysis results identified 6 high-risk genes associated with ACC, of which the mutation rates in ACC tissues were significantly higher than those in ACA or normal adrenal gland tissues, including ZNRF3, TP53, ARMC5, APC, RB1 and PRKAR1A. We proposed the concept of SHGM defined as the sum of mutated high-risk genes detected in ACC tissues, and the rates of SHGM $>0$ and SHGM $>1$ in ACC were significantly higher than those in ACA tissues, which suggest SHGM might be potential to assist the differential diagnosis of ACC with ACA, especially for ACC with small tumor size.

\section{Abbreviations}

SHGM = Sum of high-risk gene mutation

$\mathrm{ACC}=$ Adrenocortical carcinoma

ACA $=$ Adrenocortical adenoma

PUMCH=Peking Union Medical College Hospital

ENSAT $=$ European Network for the Study of Adrenal Tumors

PCR $=$ Polymerase chain reaction

FLASH $=$ Fast length adjustment of short reads to improve genome assemblies

SNVs $=$ single nucleotide variants 
Indel = insertion/ deletion

NGS = next-generation sequencing

UTR = untranslated region

\section{Declarations}

\section{Ethics approval and consent to participate}

All procedures performed in this study involving human participants were in accordance with the ethical standards of the institutional and national research committee and with the 1964 Helsinki declaration and its later amendments or comparable ethical standards. This study had been approved by the PUMCH Institutional Review Board, and the approval was papery report. We could send the electronic scanning report to the editors or reviewers if it is needed. Informed consent was obtained from all individual participants included in this study.

\section{Consent for publication}

All patients involved in this study have signed to approve the publication of their medical information.

\section{Availability of data and materials}

All the data were collected and analized by medical team of Han-Zhong Li in PUMCH. Editors and reviewers could send e-mail to hzlipumch@163.com to require any data associated with the study.

\section{Competing interests}

All of the authors declare that anyone of them has no conflict of interest.

\section{Funding}

Chinese Academy of Medical Sciences (CAMS) Initiative for Innovative Medicine (CAMS-I2M) 2017-I2M-1001.

\section{Author contributions}

Study concepts: Han-Zhong Li, Guo-Yang Zheng

Study design: Guo-Yang Zheng, Xue-Bin Zhang

Data acquisition: Guo-Yang Zheng, Yu-Shi Zhang, Jian-Hua Deng

Quality control: Xue-Bin Zhang, Yu-Shi Zhang, Xing-Cheng Wu

Data analysis and interpretation: Guo-Yang Zheng, Han-Zhong Li, Jian-Hua Deng 
Statistical analysis: Xing-Cheng Wu, Xue-Bin Zhang, Yu-Shi Zhang

Manuscript drafting: Guo-Yang Zheng, Xue-Bin Zhang, Jian-Hua Deng

Manuscript review: Han-Zhong Li, Yu-Shi Zhang, Xing-Cheng Wu

Final approval and agreement: All the authors

\section{All the authors meet the following 4 conditions:}

1. Substantial contributions to conception and design, data acquisition, or data analysis and interpretation;

2. Drafting the article or critically revising it for important intellectual content;

3. Final approval of the version to be published;

4. Agreement to be accountable for all aspects of the work in ensuring that questions related to the accuracy or integrity of the work are appropriately investigated and resolved.

\section{Acknowledgments:}

None.

\section{References}

1. Else T, Kim AC, Sabolch A, et al. Adrenocortical carcinoma. Endocr Rev. 2014;35(2):282-326. doi:10.1210/er.2013-1029.

2. Kerkhofs TM, Verhoeven RH, Van der Zwan JM, et al. Adrenocortical carcinoma: a population-based study on incidence and survival in the Netherlands since 1993. Eur J Cancer. 2013;49(11):25792586. doi:10.1016/j.ejca.2013.02.034.

3. Ayala-Ramirez M, Jasim S, Feng L, et al. Adrenocortical carcinoma: clinical outcomes and prognosis of 330 patients at a tertiary care center. Eur J Endocrinol. 2013;169(6):891-899. Published 2013 Oct 23. doi:10.1530/EJE-13-0519.

4. Fassnacht $\mathrm{M}$, Johanssen S, Fenske W, et al. Improved survival in patients with stage II adrenocortical carcinoma followed up prospectively by specialized centers. J Clin Endocrinol Metab. 2010;95(11):4925-4932. doi:10.1210/jc.2010-0803.

5. Tran TB, Liou D, Menon VG, Nissen NN. Surgical management of advanced adrenocortical carcinoma: a 21-year population-based analysis. Am Surg. 2013;79(10):1115-1118.

6. Crucitti F, Bellantone R, Ferrante A, Boscherini M, Crucitti P. The Italian Registry for Adrenal Cortical Carcinoma: analysis of a multiinstitutional series of 129 patients. The ACC Italian Registry Study Group. Surgery. 1996;119(2):161-170. doi:10.1016/s0039-6060(96)80164-4. 
7. Grubbs EG, Callender GG, Xing Y, et al. Recurrence of adrenal cortical carcinoma following resection: surgery alone can achieve results equal to surgery plus mitotane. Ann Surg Oncol. 2010;17(1):263270. doi:10.1245/s10434-009-0716-x.

8. Wein AJ. Campbell-Walsh urology. 11th ed. Philadelphia: Elsevier. 2016.1528-1576.

9. Assié $G$, Letouzé $E$, Fassnacht $M$, et al. Integrated genomic characterization of adrenocortical carcinoma. Nat Genet. 2014;46(6):607-612. doi:10.1038/ng.2953.

10. Zheng S, Cherniack AD, Dewal N, et al. Comprehensive Pan-Genomic Characterization of Adrenocortical Carcinoma [published correction appears in Cancer Cell. 2016 Aug 8;30(2):363]. Cancer Cell. 2016;29(5):723-736. doi:10.1016/j.ccell.2016.04.002.

11. Fassnacht $M$, Libé R, Kroiss M, Allolio B. Adrenocortical carcinoma: a clinician's update. Nat Rev Endocrinol. 2011;7(6):323-335. doi:10.1038/nrendo.2010.235.

12. Bilimoria KY, Shen WT, Elaraj D, et al. Adrenocortical carcinoma in the United States: treatment utilization and prognostic factors. Cancer. 2008;113(11):3130-3136. doi:10.1002/cncr.23886.

13. Terzolo M, Angeli A, Fassnacht $\mathrm{M}$, et al. Adjuvant mitotane treatment for adrenocortical carcinoma. $N$ Engl J Med. 2007;356(23):2372-2380. doi:10.1056/NEJMoa063360.

14. Arola J, Liu J, Heikkilä P, et al. Expression of inhibin alpha in adrenocortical tumours reflects the hormonal status of the neoplasm. J Endocrinol. 2000;165(2):223-229. doi:10.1677/joe.0.1650223.

15. Jorda M, De MB, Nadji M. Calretinin and inhibin are useful in separating adrenocortical neoplasms from pheochromocytomas. Appl Immunohistochem Mol Morphol. 2002;10(1):67-70. doi:10.1097/00129039-200203000-00012.

16. Ghorab Z, Jorda M, Ganjei P, Nadji M. Melan A (A103) is expressed in adrenocortical neoplasms but not in renal cell and hepatocellular carcinomas. Appl Immunohistochem Mol Morphol. 2003;11(4):330-333. doi:10.1097/00129039-200312000-00009.

17. Duregon E, Volante M, Giorcelli J, Terzolo M, Lalli E, Papotti M. Diagnostic and prognostic role of steroidogenic factor 1 in adrenocortical carcinoma: a validation study focusing on clinical and pathologic correlates. Hum Pathol. 2013;44(5):822-828. doi:10.1016/j.humpath.2012.07.025.

18. Aubert S, Wacrenier A, Leroy $X$, et al. Weiss system revisited: a clinicopathologic and immunohistochemical study of 49 adrenocortical tumors. Am J Surg Pathol. 2002;26(12):16121619. doi:10.1097/00000478-200212000-00009.

19. Libé R. Adrenocortical carcinoma (ACC): diagnosis, prognosis, and treatment. Front Cell Dev Biol. 2015;3:45. Published 2015 Jul 3. doi:10.3389/fcell.2015.00045.

20. Morimoto R, Satoh F, Murakami O, et al. Immunohistochemistry of a proliferation marker Ki67/MIB1 in adrenocortical carcinomas: Ki67/MIB1 labeling index is a predictor for recurrence of adrenocortical carcinomas. Endocr J. 2008;55(1):49-55. doi:10.1507/endocrj.k07-079.

21. Papotti M, Libè R, Duregon E, Volante M, Bertherat J, Tissier F. The Weiss score and beyond-histopathology for adrenocortical carcinoma. Horm Cancer. 2011;2(6):333-340. doi:10.1007/s12672011-0088-0. 
22. Mihai R. Diagnosis, treatment and outcome of adrenocortical cancer. Br J Surg. 2015;102(4):291306. doi:10.1002/bjs. 9743 .

23. Tissier F, Cavard C, Groussin L, et al. Mutations of beta-catenin in adrenocortical tumors: activation of the Wnt signaling pathway is a frequent event in both benign and malignant adrenocortical tumors. Cancer Res. 2005;65(17):7622-7627. doi:10.1158/0008-5472.CAN-05-0593.

24. Kjellman M, Roshani L, Teh BT, et al. Genotyping of adrenocortical tumors: very frequent deletions of the MEN1 locus in 11q13 and of a 1-centimorgan region in 2p16. J Clin Endocrinol Metab. 1999;84(2):730-735. doi:10.1210/jcem.84.2.5506.

25. Barzon L, Chilosi M, Fallo F, et al. Molecular analysis of CDKN1C and TP53 in sporadic adrenal tumors. Eur J Endocrinol. 2001;145(2):207-212. doi:10.1530/eje.0.1450207.

26. Nobori T, Miura K, Wu DJ, Lois A, Takabayashi K, Carson DA. Deletions of the cyclin-dependent kinase-4 inhibitor gene in multiple human cancers. Nature. 1994;368(6473):753-756. doi:10.1038/368753a0.

27. Gaujoux S, Tissier F, Groussin L, et al. Wnt/beta-catenin and 3',5'-cyclic adenosine 5'monophosphate/protein kinase A signaling pathways alterations and somatic beta-catenin gene mutations in the progression of adrenocortical tumors. J Clin Endocrinol Metab. 2008;93(10):41354140. doi:10.1210/jc.2008-0631.

28. Juhlin CC, Goh G, Healy JM, et al. Whole-exome sequencing characterizes the landscape of somatic mutations and copy number alterations in adrenocortical carcinoma. J Clin Endocrinol Metab. 2015;100(3):E493-E502. doi:10.1210/jc.2014-3282.

29. Zhang Q, Cui L, Gao JP, et al. Whole-genome sequencing revealed armadillo repeat containing 5 (ARMC5) mutation in a Chinese family with ACTH-independent macronodular adrenal hyperplasia. Endocr J. 2018;65(3):269-279. doi:10.1507/endocrj.EJ17-0317.

\section{Figures}




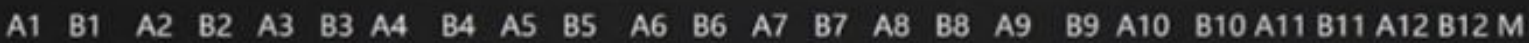

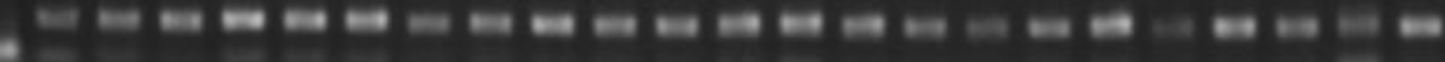

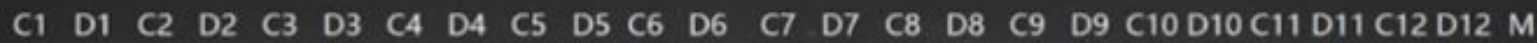

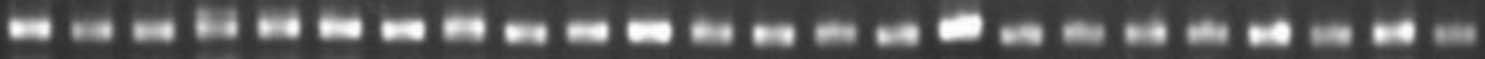
(a)

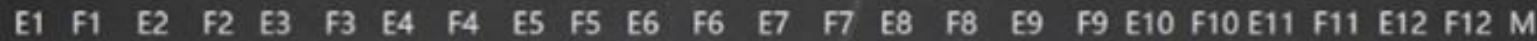

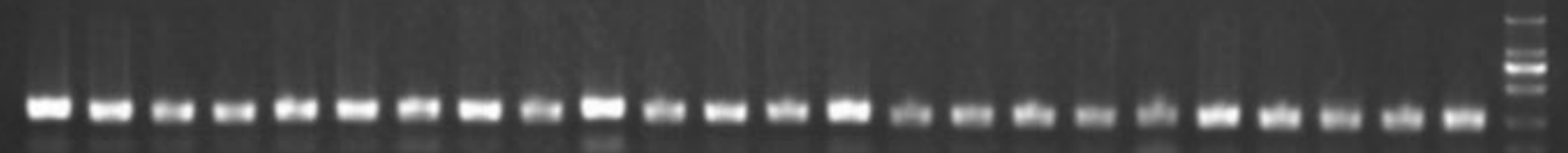

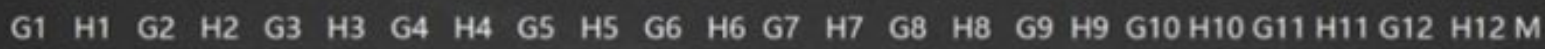

\section{Figure 1}

Multiple PCR results of 94 cases of eligible samples for quality control. A1 for negative control, A2 for positive control, M for DL2000Marker. 
1600

1400

1200

1000

800

600

400

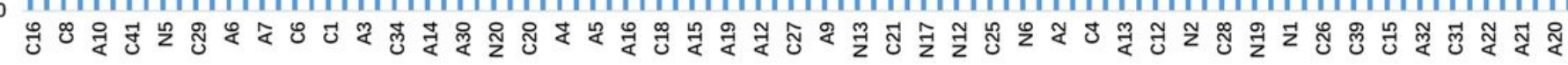

Figure 2

Sequencing depth of all 94 samples for quality analysis. Ranged from $190 \mathrm{X}$ to $1536 \mathrm{X}$, and the mean was 733X.

$$
>10 X
$$

1.2

1

0.8

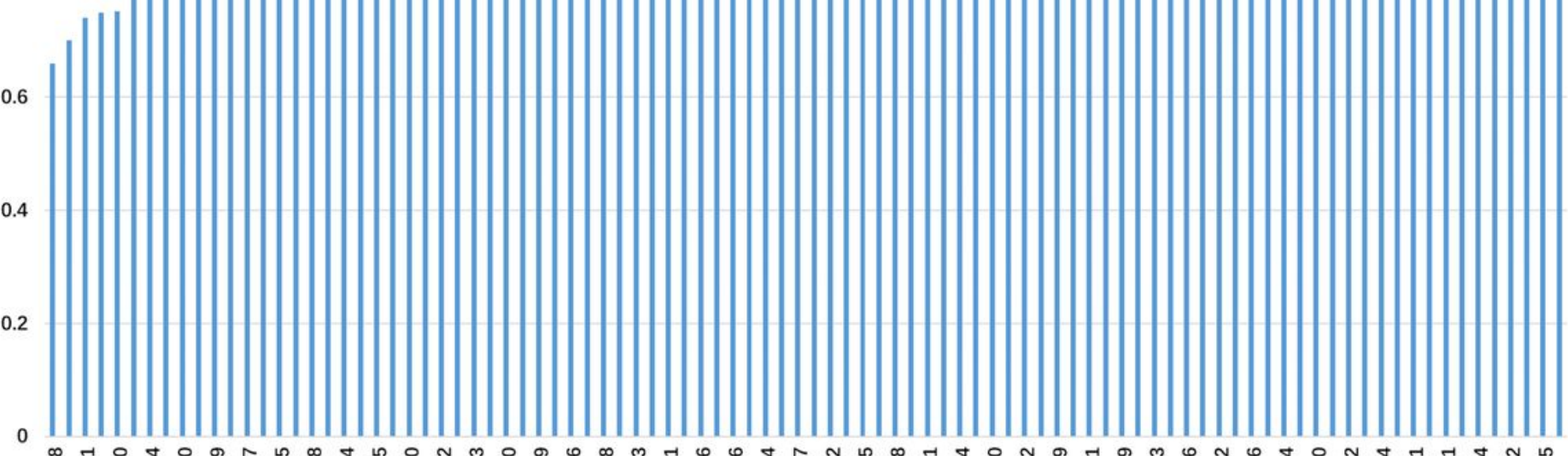

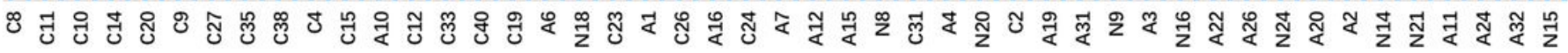

Figure 3

Sequencing depth of target gene fragments was above 10X in $95.1 \%$ samples. 

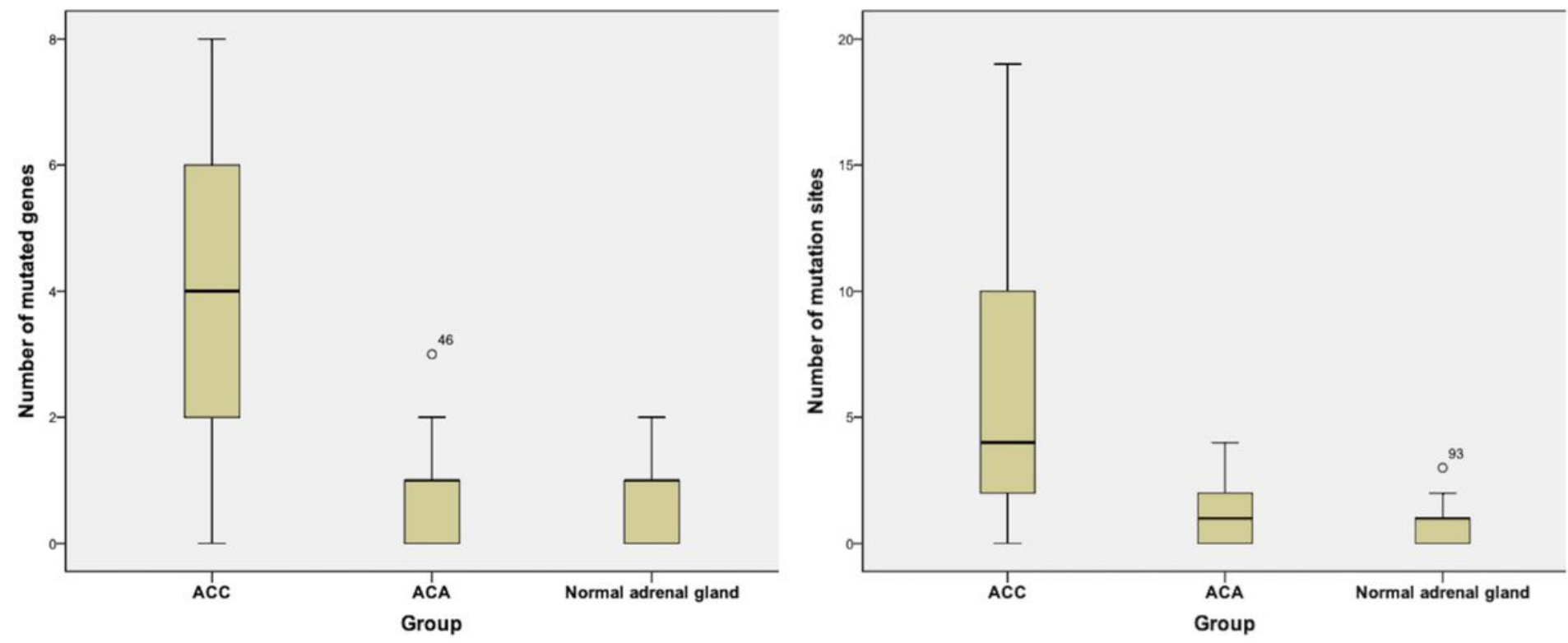

Figure 4

Kruskal-Wallis test box-plot of mutated target genes and mutation sites in 3 groups of tissues.

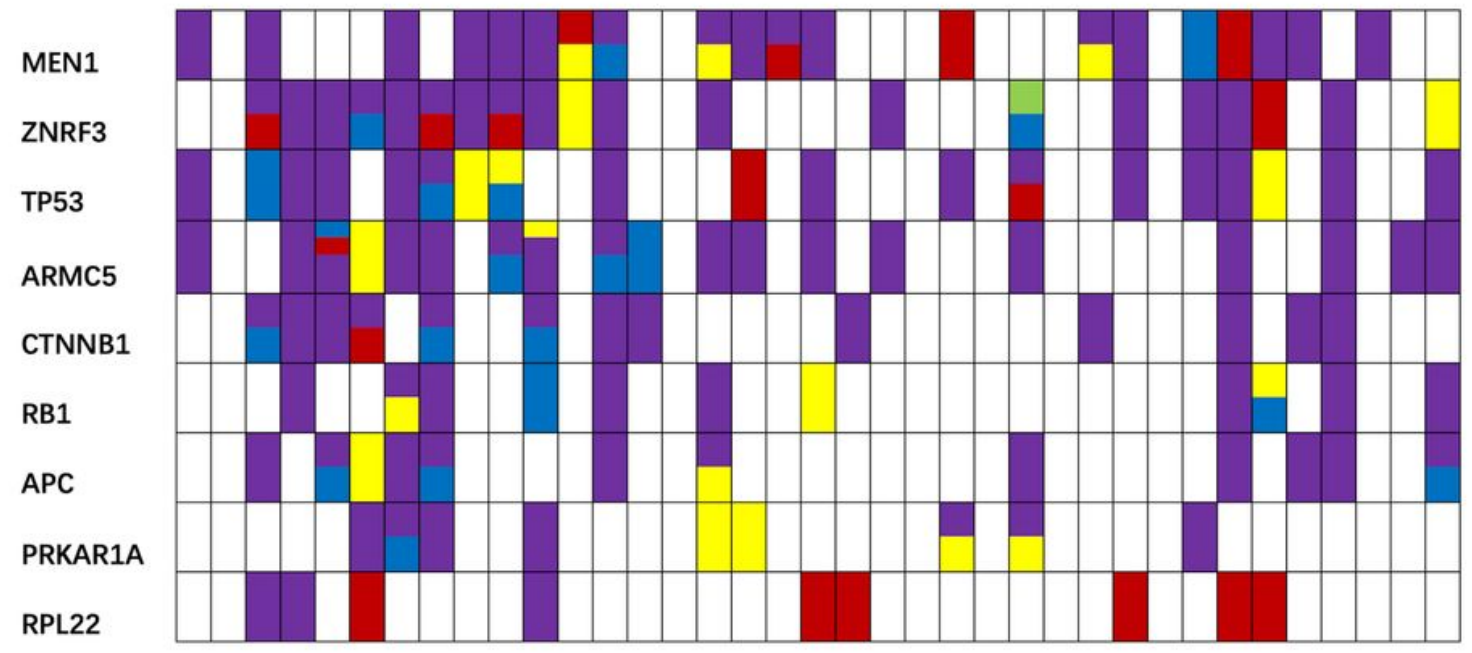

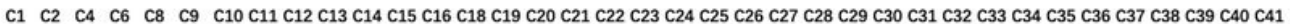

\section{Figure 5}

The mutation information of nine target genes in ACC tissues. 


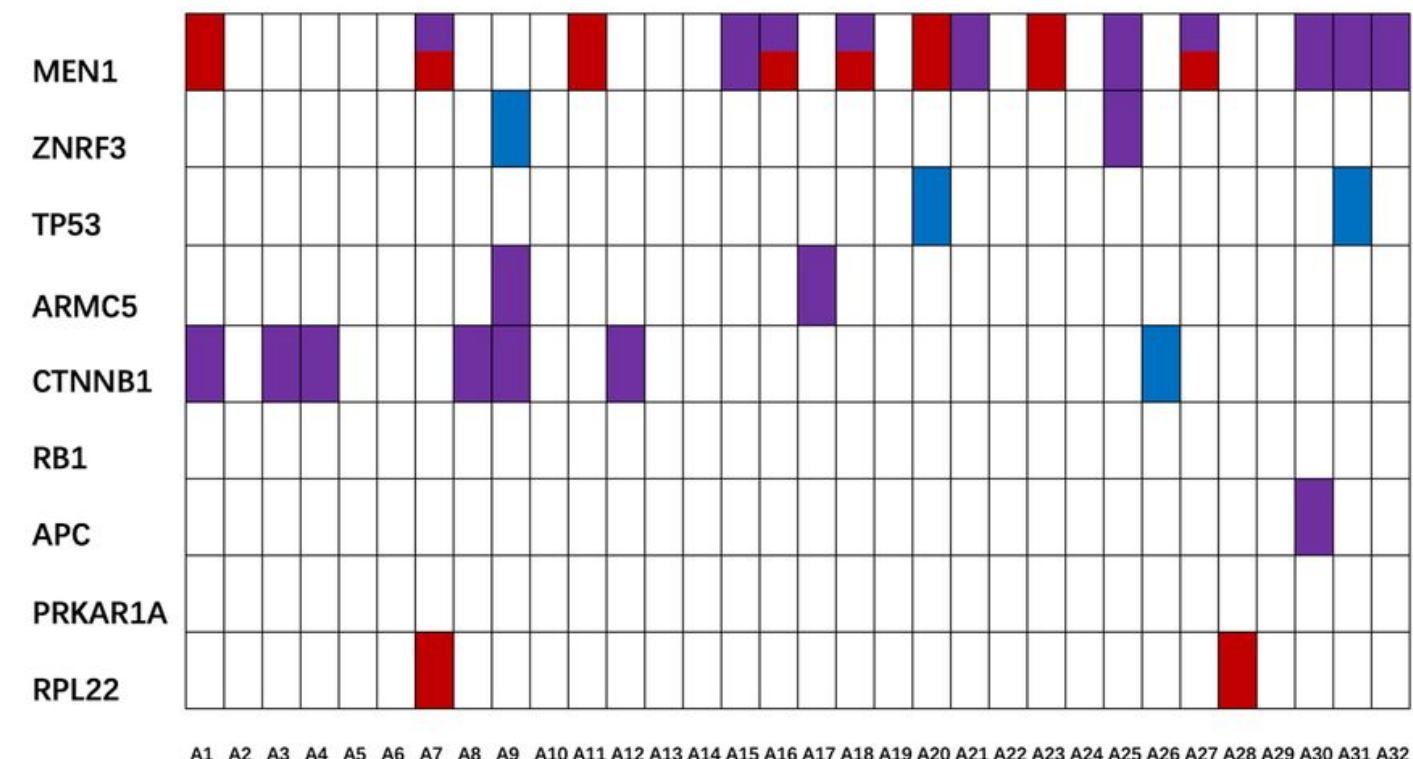

A1 A2 A3 A4 A5 A6 A7 A8 A9 A10 A11 A12 A13 A14 A15 A16 A17 A18 A19 A20 A21 A22 A23 A24 A25 A26 A27 A28 A29 A30 A31 A32

\section{Figure 6}

The mutation information of nine target genes in ACA tissues.

MEN1

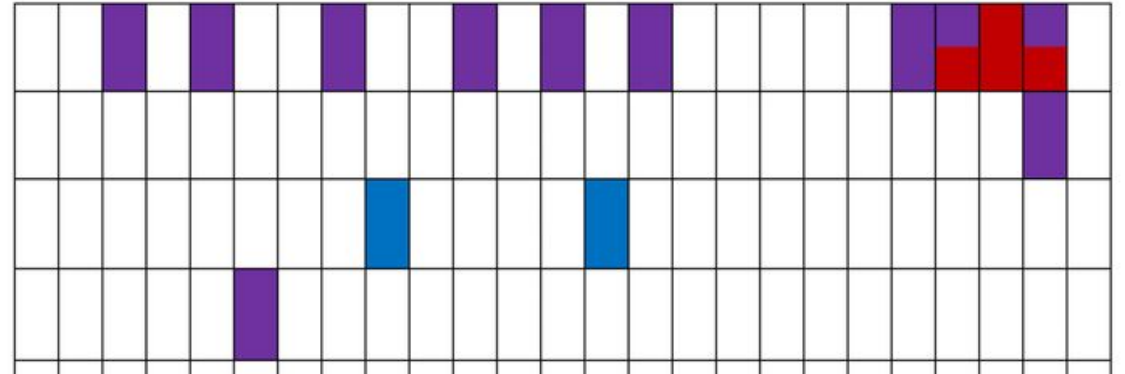

ARMC5

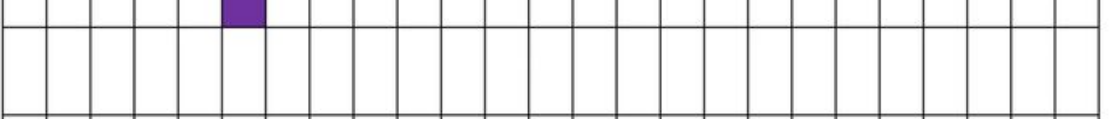

CTNNB1

RB1

APC

PRKAR1A

RPL22

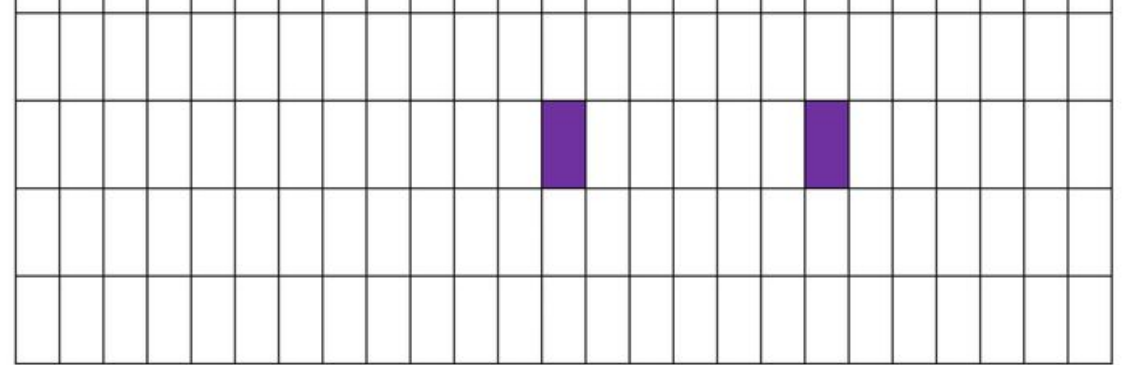

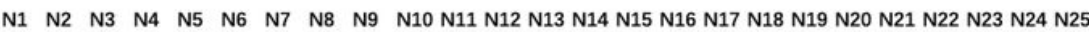

Figure 7

The mutation information of nine target genes in normal adrenal gland tissues. 